\title{
Saturable nonlinear dielectric waveguide with applications to broad-area semiconductor lasers
}

\author{
David Mehuys, Michael Mittelstein, Joseph Salzman,* and Amnon Yariv \\ Department of Applied Physics, 128-95, California Institute of Technology, Pasadena California 91125
}

Received July 6, 1987; accepted August 4, 1987

\begin{abstract}
Self-focusing in a passive dielectric waveguide with a saturable nonlinearity is studied. The eigensolutions constitute a good approximation to the lateral modes of broad-area semiconductor lasers under low-duty-cycle pulsed conditions. The laser modes are predicted to consist of adjacent filaments coupled in phase, leading to a single-lobed far field, and to be stable with increased current injection above saturation intensity. The ultimate filament spacing is inversely proportional to the threshold gain, and thus wider filaments are expected in lowthreshold broad-area lasers.
\end{abstract}

Broad-area semiconductor lasers are candidates for high-power coherent sources when pumped to levels far beyond threshold. Recently, high-quality broadarea lasers were demonstrated to oscillate coherently into a nearly diffraction-limited single-lobed far-field pattern ${ }^{1,2}$ and were injection locked. ${ }^{3}$ In all cases, the near field is characterized by a small ripple (10-20\%) superimposed upon a large dc amplitude and is stable with increased injection current. These modes have commonly been referred to as higher-order HermiteGaussian. However, this description is inconsistent with the observed single-lobed far fields. An alternative interpretation based on Thompson's treatment of self-focusing in semiconductor lasers ${ }^{4}$ was recently suggested.5,6 According to that, these modes consist of coupled filaments. However, in the high-power regime, both below-threshold linear theories and nearthreshold Kerr-law nonlinearity models are not applicable. In this Letter we characterize solutions in a passive dielectric medium with saturable nonlinearity and discuss the implications for broad-area semiconductor lasers.

The nonlinearity in the field equation of a broadarea semiconductor laser arises from the carrier dependence of the refractive index. In the pumped region, the carrier density is not uniform across the device width but is inversely related to the local photon density. This is a consequence of gain saturation and can be seen directly from the spatial rate equation. ${ }^{4}$ Although the gain is locally depressed by stimulated emission, the refractive index is locally increased by the band-edge effect. Thus the nonlinearity is of the self-focusing type.

By linearizing the gain-current relation about the threshold gain and using an effective index approximation, ${ }^{7}$ the carrier-dependent dielectric constant in a semiconductor laser is described as ${ }^{8}$

$$
n_{\mathrm{eff}}^{2}=n_{0}^{2}+\epsilon_{\mathrm{sat}}\left(1+\frac{i}{b}\right) \frac{\frac{|E|^{2}}{E_{\mathrm{sat}}^{2}}-\frac{J-J_{\mathrm{th}}}{J_{\mathrm{sat}}}}{1+\frac{|E|^{2}}{E_{\mathrm{sat}}^{2}}},
$$

where $n_{0}^{2}$ is the effective index of refraction at threshold carrier density, $\epsilon_{\mathrm{sat}}=n_{\mathrm{tr}}^{2}-n_{0}^{2}$ is the difference in dielectric constant between threshold carrier density and transparency carrier density, $b$ is the antiguiding factor, $E(x)$ is the lateral electric field, and $E_{\text {sat }}^{2}$ is the saturation intensity. The term $\left(J-J_{\text {th }}\right) / J_{\text {sat }}$ represents the current pumping above threshold in units of the saturation current density; for convenience we refer to this term hereafter as $\beta$. The full treatment of the lateral semiconductor laser modes with complex nonlinear index [Eq. (1)] will be presented elsewhere. ${ }^{9}$ Here we describe the solutions of a real-index dielectric waveguide with saturable nonlinearity. These solutions, since they are simpler to analyze, enable us to draw some conclusions about the modes of a semiconductor laser in the saturated regime and to identify the important physical parameters.

The normalized electric field $e(x) \equiv E(x) / \sqrt{\beta} E_{\text {sat }}$ satisfies the nonlinear wave equation

$$
\frac{1}{k_{0}^{2}} \frac{\mathrm{d}^{2} e}{\mathrm{~d} x^{2}}+\left[n_{0}^{2}+\frac{\alpha\left(e^{2}-1\right)}{1+\beta e^{2}}-\eta^{2}\right] e=0,
$$

where $k_{0}$ is the free-space propagation constant, $\eta$ is the eigenindex, and $\alpha=\beta \epsilon_{\mathrm{sat}}$. Thus the local index of refraction is increased proportional to the optical intensity when $\alpha>0$ (self-focusing).

By using the intensity-dependent real dielectric constant of Eq. (2) instead of Eq. (1), we neglect phase variations across the laser. Although broad-area lasers are globally gain guided, it is the real part of the refractive-index profile that governs the filamentary behavior in regions of high injected current density. Therefore the solutions of Eq. (2) represent good approximations to the mode amplitude of a real laser.

Equation (2) has the first integral

$$
\begin{aligned}
\left(\frac{1}{k_{0}} \frac{\mathrm{d} e}{\mathrm{~d} x}\right)^{2}+ & \left(n_{0}^{2}-\eta^{2}\right) e^{2} \\
& +\epsilon_{\mathrm{sat}} e^{2}\left[1-(1+\beta) \frac{\ln \left(1+\beta e^{2}\right)}{\beta e^{2}}\right]=C,
\end{aligned}
$$


where $C$ is a real constant. For the case of $\beta=0$, the nonlinear term is $1 / 2 \alpha e^{4}$ and solutions are the Jacobian elliptic functions $c n(x)$ and $d n(x)$. The solutions of Eq. (2) are analogous functions. The case $C=0$ corresponds to the Kerr-law single-filament solution since from Eq. (3) we have $e, \mathrm{~d} e / \mathrm{d} x=0$ at $x= \pm \infty$ (bounded solutions). Figure 1(a) illustrates the normalized eigenvalue detuning $\left(n_{0}^{2}-\eta^{2}\right) / \alpha$ as a function of pumping $\beta$ for this case. The case $C<0$ corresponds to periodic solutions, which are unipolar (adjacent filaments coupled in phase). Similar to the dnoidal functions, these solutions exhibit a single-lobed far-field pattern. The case $C>0$ corresponds to the bipolar cnoidal functions (adjacent filaments coupled $\pi$ rad out of phase) and have double-lobed far fields. Figure 1(b) illustrates representative solutions with $C<0, C$ $=0$, and $C>0$ for a current-pumping value $\beta=1$. The value of $C$ appropriate for a given waveguide is determined by the boundary conditions, as we show next.

Consider a three-layer lateral waveguide with effective index $n_{0}^{2}$ in the inner (or core) region and $n_{1}^{2}$ in the outer (or cladding) regions. In stripe-geometry semiconductor lasers, the unpumped outer regions are lossy. Thus light is absorbed here-producing carri-
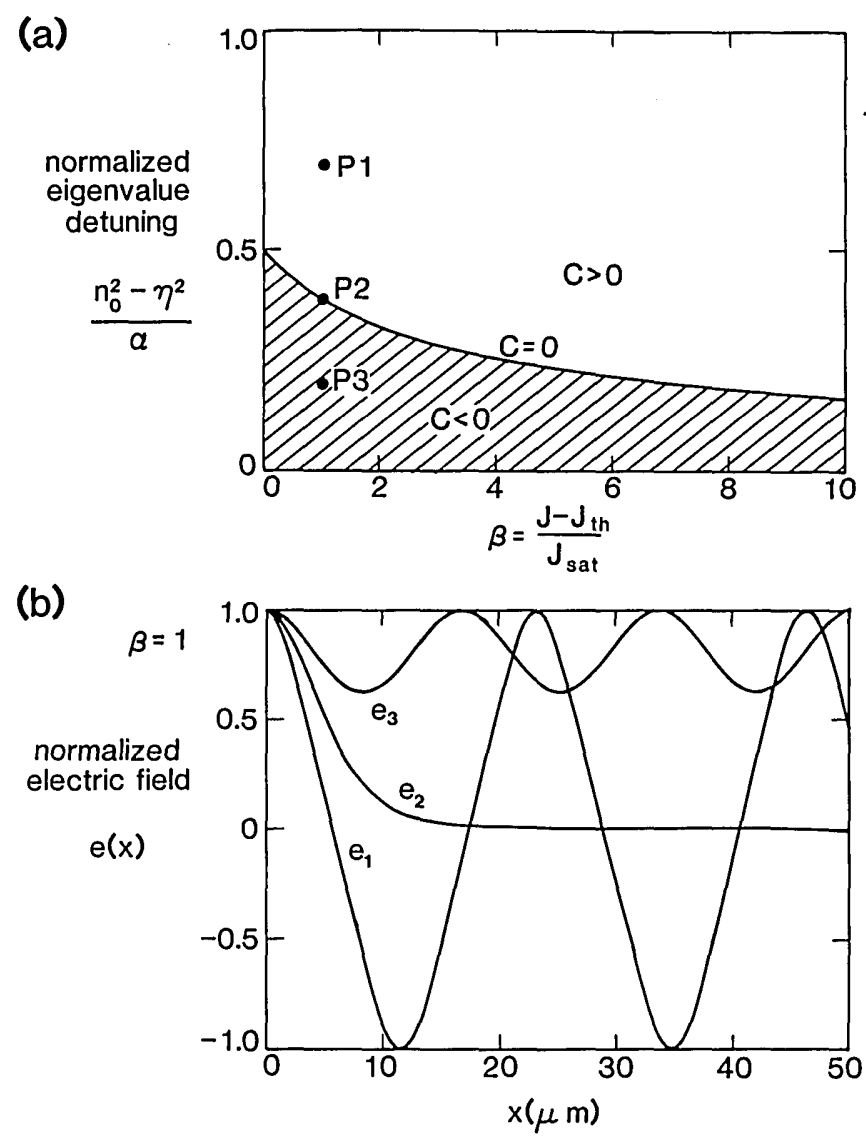

Fig. 1. (a) The normalized eigenvalue detuning $\left(n_{0}^{2}-\eta^{2}\right) / \alpha$ as a function of current pumping parameter $\beta$ for the singlefilament solution $(C=0)$. The regions of $C>0$ and $C<0$ (hatched) are also indicated. (b) Representative solutions for the cases $C<0, C=0$, and $C>0$ are shown. The curves $\mathrm{e}_{1}-\mathrm{e}_{3}$ correspond to the points P1-P3 in (a). ers and decreasing the local index of refraction-and so a self-defocusing nonlinearity is appropriate. The intensity-dependent effective index is modeled as in the pumped inner region but with $\alpha \rightarrow \alpha^{\prime}$, where $\alpha^{\prime} / \beta=$ $n_{1}^{2}-n_{\mathrm{tr}}^{2}$. The first integral must be characterized by integration constant $C^{\prime}=0$ in the outer region. Matching $e$ and $\mathrm{d} e / \mathrm{d} x$ at the interfaces gives the following expression for $C$ in the pumped region:

$$
\begin{aligned}
C & =\int_{0}^{I} n_{0}^{2}(I)-n_{1}^{2}(I) \mathrm{d} I \\
& =\left(n_{0}^{2}-n_{1}^{2}\right)\left[1+\beta \frac{\alpha}{\alpha+\alpha^{\prime}}\right] \ln (1+\beta I)^{1 / \beta},
\end{aligned}
$$

where $I$ is the value of $e^{2}$ at the interface $(I \geq 0)$. Thus the sign of $C$ is determined by the refractive-index step at the interface. In gain-guided semiconductor lasers, the carrier dependence reduces the refractive index in the pumped region relative to the unpumped region. Therefore, if no deliberate index step is introduced in the laser design, we have $n_{0}^{2}-n_{1}^{2}<0$. Thus $C<0$, and we expect the in-phase nonlinear mode to be favored, with a resulting single-lobed far field.

This model considers only the band-edge contribution to the refractive index. Under $\mathrm{cw}$ operation or particularly high current pumping, heating effects must also be considered. ${ }^{10}$ Since $\mathrm{d} n / \mathrm{d} T>0$, the refractive index of the pumped regions may surpass that of the unpumped regions. Under such conditions, the out-of-phase nonlinear mode would be favored. Still, we expect the in-phase mode to be preferred under low-duty-cycle pulsed operation.

Below saturation (in the Kerr nonlinear regime), the filament spacing decreases with increasing field intensity. However, since the nonlinearity is saturable, this process is self-stabilized as the pump level $\beta \rightarrow \infty$. In particular, the periodicity of the $C<0$ solution approaches $2 \epsilon_{\text {sat }}$ in the small-modulation limit. In the linearized gain approximation, $\epsilon_{\text {sat }} \simeq\left(n_{0} / k_{0}\right) b G_{\text {th }}$, where $b$ is the antiguiding factor mentioned above and $G_{\text {th }}$ is the threshold gain. Typically, $\epsilon_{\text {sat }} \gg n_{0}^{2}-\eta^{2}$ (the eigenvalue detuning), and the saturated filament spacing, $\Delta x_{\text {sat }}$, is

$$
\Delta x_{\mathrm{sat}}=\frac{\lambda}{\left(2 \frac{n_{0}}{k_{0}} b G_{\mathrm{th}}\right)^{1 / 2}},
$$

where $\lambda$ is the free-space wavelength. Thus wider filaments are expected in low-threshold lasers. Estimated parameters for our graded-index separate-heterostructure single-quantum-well wafers are $b=2$ and $G_{\text {th }}=30 \mathrm{~cm}^{-1}$, which at a wavelength of $\lambda=845 \mathrm{~nm}$ gives $\Delta x_{\text {sat }} \simeq 12 \mu \mathrm{m}$. This compares favorably with the observed spacing of $9-10 \mu \mathrm{m} .^{1}$

Finally, we comment on the effect of excluding gain from the calculation. In an active medium, self-consistency requires local phase fronts that are retarded where the optical intensity is high, thereby directing 
photons into the high-intensity region and compensating for the locally depressed gain. Thus the phase front is modulated in antiphase to the intensity ripple. In addition, since the mode is confined by the outer lossy regions, an overall phase-front curvature is required to direct the photons outward. This effect introduces a slowly varying component into the amplitude equation and causes the local filament spacing to decrease toward the interface. ${ }^{9}$

In conclusion, we have presented solutions of the nonlinear field equation in a passive dielectric waveguide with a saturable self-focusing nonlinearity. Solutions with adjacent filaments coupled $\pi$ rad out of phase are ruled out by the boundary conditions prevalent in gain-guided broad-area semiconductor lasers operated under low-duty-cycle pulsed conditions. The in-phase solutions are stable above saturation intensity, and the saturated filament spacing is proportional to the refractive-index difference between threshold and transparency carrier densities. The observed single-lobed far fields and near-field stability can be accounted for by this analysis.

The research described in this Letter was conducted in the Department of Applied Physics, California Institute of Technology, with the support of the U.S. Office of Naval Research and the National Science Foundation.
* Present address, Bell Communications Research, 331 Newman Springs Road, Red Bank, New Jersey 07701-7020.

\section{References}

1. A. Larsson, M. Mittelstein, Y. Arakawa, and A. Yariv, Electron. Lett. 22, (1986).

2. M. Sakamoto and Y. Kato, Appl. Phys. Lett. 50, 869 (1987).

3. M. M. Leopold, R. G. Podgornik, and R. A. Williams, Electron. Lett. 22, 1224 (1986).

4. G. H. B. Thompson, Optoelectronics 4, 257 (1972).

5. A. Larsson, J. Salzman, M. Mittelstein, and A. Yariv, J. Appl. Phys. 60, 66 (1986).

6. J. Salzman, A. Larsson, and A. Yariv, Appl. Phys. Lett. 49, 611 (1986).

7. W. Streifer and E. Kapon, Appl. Opt. 18, 3724 (1979).

8. D. Mehuys, M. Mittelstein, R. J. Lang, J. Salzman, and A. Yariv, in Digest of Conference on Lasers and ElectroOptics (Optical Society of America, Washington, D.C., 1987), paper TuK27.

9. D. Mehuys, R. J. Lang, M. Mittelstein, J. Salzman, and A. Yariv, "Self-stabilized nonlinear lateral modes of broad area lasers," IEEE J. Quantum Electron. (to be published).

10. G. R. Hadley, J. P. Hohimer, and A. Owyoung, in Digest of Conference on Lasers and Electro-Optics (Optical Society of America, Washington, D.C., 1987), paper FD1. 